manuscript No.

(will be inserted by the editor)

\title{
Global sensitivity analysis of stochastic computer models with joint metamodels
}

\author{
Bertrand Iooss · Mathieu Ribatet · Amandine Marrel
}

Received: date / Accepted: date

\begin{abstract}
The global sensitivity analysis method, used to quantify the influence of uncertain input variables on the response variability of a numerical model, is applicable to deterministic computer code (for which the same set of input variables gives always the same output value). This paper proposes a global sensitivity analysis methodology for stochastic computer code (having a variability induced by some uncontrollable variables). The framework of the joint modeling of the mean and dispersion of heteroscedastic data is used. To deal with the complexity of computer experiment outputs, non parametric joint models (based on Generalized Additive Models and Gaussian processes) are discussed. The relevance of these new models is analyzed in terms of the obtained variance-based sensitivity indices with two case studies. Results show that the joint modeling approach leads accurate sensitivity index estimations even when clear heteroscedasticity is present.
\end{abstract}

Keywords Computer experiment - Generalized additive model $\cdot$ Gaussian process $\cdot$ Joint modeling $\cdot$ Sobol indices . Uncertainty

\section{B. Iooss}

CEA, DEN, Centre de Cadarache, F-13108 Saint Paul lez Durance, France

Tel.: +33-4-42257273

Fax: +33-4-42252408

E-mail: bertrand.iooss@cea.fr

M. Ribatet

Ecole Polytechnique Fédérale de Lausanne, Institute of Mathematics, Lausanne, Switzerland

A. Marrel

CEA, DEN, F-13108 Saint Paul lez Durance, France

Present address: Institut Français du Pétrole, 92852 Rueil-Malmaison, France

\section{Introduction}

Many phenomena are modeled by mathematical equations which are implemented and solved by complex computer code. These computer models often take as inputs a high number of numerical variables and physical variables, and give several outputs (scalars or functions). For the development of such computer models, its analysis, or its use, the global Sensitivity Analysis (SA) method is an invaluable tool (Saltelli et al., 2000; Kleijnen, 1997; Helton et al., 2006). It takes into account all the variation ranges of the inputs, and tries to apportion the output uncertainty to the uncertainty in the input factors. These techniques, often based on the probabilistic framework and Monte-Carlo methods, require a lot of simulations. The uncertain input variables are modeled by random variables and characterized by their probabilistic density functions. The SA methods are used for model calibration (Kennedy and O'Hagan, 2001), model validation (Bayarii et al.,2007), decision making process (De Rocquigny et al., 2008), i.e. all the processes where it is useful to know which variables mostly contribute to output variability .

The current SA methods are applicable to the deterministic computer code, i.e. for which the same set of input variables always gives the same output values. The randomness is limited to the model inputs, whereas the model itself is deterministic. For example in the nuclear engineering domain, global sensitivity analysis tools have been applied to waste storage safety studies (Helton et al., 2006), and pollutant transport modeling in the aquifer (Volkova et al., 2008). In such industrial studies, numerical models are often too time consuming for applying directly the global SA methods. To avoid this problem, one solution consists in replacing the time consuming computer code by an approximate mathematical model, called metamodel (Sacks et al., 1989; Fang et al., 2006). This function must be as representative as possible of the computer code, with good prediction capabil- 
ities and must require a negligible calculation time. Several metamodels are classically used: polynomials, splines, neural networks, Gaussian processes (Chen et al., 2006; Fang et al., 2006).

In this paper, we are not concerned by deterministic computer models but by stochastic numerical models - i.e. when the same input variables set leads to different output values. The model itself relies on probabilistic methods (e.g. MonteCarlo) and is therefore intrinsically stochastic because of some "uncontrollable variables". For the uncertainty analysis, Kleijnen (1997) has raised this question, giving an example concerning a queueing model. In the nuclear engineering domain, examples are given by Monte-Carlo neutronic models used to calculate elementary particles trajectories, Lagrangian stochastic models for simulating a large number of particles inside turbulent media (in atmospheric or hydraulic environment). In our study, "uncontrollable" variables correspond to variables that are known to exist, but unobservable, inaccessible or non describable for some reasons. It includes the important case in which observable vector variables are too complex to be described or synthesized by a reasonable number of scalar parameters. This last situation might concern the code for which some simulations of complex random processes are used. For example, one can quote some partial differential equation resolutions in heterogeneous random media simulated by geostatistical techniques (e.g. fluid flows in oil reservoirs, Zabalza et al., 1998, and acoustical wave propagation in turbulent fluids, Iooss et al., 2002), where the uncontrollable variable is the simulated spatial field involving several thousand scalar values for each realization. Of course, in this case, behind this uncontrollable variable stands a fully controllable parameter: the random seed. However, the effect of the random seed on the computer code output is totally chaotic because a slight modification of the random seed leads to a very different random medium realization. For simplicity and for generality, we use the expression "uncontrollable variable" in this paper.

For stochastic computer models, classical metamodels (devoted to approximate deterministic computer models) are not pertinent anymore. To overcome this problem, the commonly used Gaussian process (Gp) model (Sacks et al., 1989; Marrel et al., 2008) can include an additive error component (called the "nugget effect") by adding a constant term into its covariance function. However, it supposes that the error term is independent of the input variables (homoscedasticity hypothesis), which means that the uncontrollable variable does not interact with controllable variables. This hypothesis limits the use of $\mathrm{Gp}$ to specific cases even if recently, some authors (e.g. Kleijnen and van Beers, 2005) demonstrated the usefulness of Gp for stochastic computer model in heteroscedastic cases.
To construct heteroscedastic metamodels for stochastic computer code, Zabalza et al. (1998) have proposed another approach by modeling the mean and the dispersion (i.e. the variance) of computer code outputs by two interlinked Generalized Linear Models (GLMs). This approach, called the joint model, has been previously studied in the context of experimental data modeling (Smyth, 1989; McCullagh and Nelder, 1989).

Modeling the mean and variance of a response variable in function of some controllable variables is of primary concern in product development and quality engineering methods (Phadke, 1989): experimentation is used to determine the factor levels so that the product is insensitive to potential variations of environmental conditions. This can be summarized, in the framework of the robust design, as the optimization of a mean response function while minimizing a variance function. In this context, Vining and Myers (1990) propose to build polynomial models for the mean and the variance separately, while Lee and Nelder (2003) consider the joint GLM approach. A recent and complete review on this subject can be found in Bursztyn and Steinberg (2006). Dealing with computer experiments instead of physical ones, Bates et al. (2006) propose different strategies for designing and analyzing robust design experiments. In this case, the noise factors are fully controllable. This allows the authors to provide a powerful stochastic emulator strategy.

Following the work of Zabalza et al. (1998), Iooss and Ribatet (2006, 2009) have recently introduced the joint model to perform a global sensitivity analysis of a stochastic computer code. Results show that a total sensitivity index of all the uncontrollable variables can be computed using the dispersion component of the joint model. However, the parametric form of the GLM framework provides some limitations when modeling complex computer code outputs. To bypass this hurdle, this paper suggests the use of non parametric models. Due to its similarity with GLMs, Generalized Additive Models (GAM) are considered (Hastie and Tibshirani, 1990; Wood and Augustin, 2002), even though Gp or other non parametric models should also be a relevant solution.

This paper starts by describing the joint model construction, firstly with the GLM, secondly with the GAM. We will also show how other models, like Gp, can be used to model the mean and dispersion components. The third section describes the global sensitivity analysis for deterministic models, and its extension to stochastic models using joint models. Particular attention is devoted to the calculation of variance-based sensitivity indices (the so-called Sobol indices). Considering a simple analytic function, the performance of the proposed approach is compared to other commonly used models. Next, an application on an actual industrial case (groundwater radionuclide migration modeling) is given. Finally, some conclusions synthesize the contributions of this work. 


\section{Joint modeling of mean and dispersion}

\subsection{Using the Generalized Linear Models}

The class of GLM allows to extend the class of the traditional linear models by the use of: (a) a distribution which belongs to the exponential family; (b) and a link function which connects the explanatory variables to the explained variable (Nelder and Wedderburn, 1972). Let us describe the first component of the model concerning the mean:

$\begin{cases}\mathbb{E}\left(Y_{i}\right) & =\mu_{i}, \quad \eta_{i}=g\left(\mu_{i}\right)=\sum_{j} x_{i j} \beta_{j} \\ \operatorname{Var}\left(Y_{i}\right) & =\phi_{i} v\left(\mu_{i}\right)\end{cases}$

where $\left(Y_{i}\right)_{i=1 \ldots n}$ are independent random variables with mean $\mu_{i} ; x_{i j}$ are the observations of the variable $X_{j} ; \beta_{j}$ are the regression parameters which have to be estimated; $\eta_{i}$ is the mean linear predictor; $g(\cdot)$ is a differentiable monotonous function (called the link function); $\phi_{i}$ is the dispersion parameter and $v(\cdot)$ is the variance function. To estimate the mean component, the functions $g(\cdot)$ and $v(\cdot)$ have to be specified. Some examples of link functions are given by the identity (traditional linear model), root square, logarithm, and inverse functions. Some examples of variance functions are given by the constant (traditional linear model), identity and square functions.

Within the joint model framework, the dispersion parameter $\phi_{i}$ is not supposed to be constant as in a traditional GLM, but is supposed to vary according to the model:

$\begin{cases}\mathbb{E}\left(d_{i}\right) & =\phi_{i}, \quad \zeta_{i}=h\left(\phi_{i}\right)=\sum_{j} u_{i j} \gamma_{j}, \\ \operatorname{Var}\left(d_{i}\right) & =\tau v_{d}\left(\phi_{i}\right),\end{cases}$

where $d_{i}$ is a statistic representative of the dispersion, $\gamma_{j}$ are the regression parameters which have to be estimated, $h(\cdot)$ is the dispersion link function, $\zeta_{i}$ is the dispersion linear predictor, $\tau$ is a constant and $v_{d}(\cdot)$ is the dispersion variance function. $u_{i j}$ are the observations of the explanatory variable $U_{j}$. The variables $\left(U_{j}\right)$ are generally taken among the explanatory variables of the mean $\left(X_{j}\right)$, but might differ. To ensure positivity, $h(\phi)=\log \phi$ is often taken for the dispersion link function while the statistic $d$ representing the dispersion is generally taken to be the deviance contribution which is approximately $\chi^{2}$ distributed. Therefore, as the $\chi^{2}$ distribution is a particular case of the Gamma distribution, $v_{d}(\phi)=\phi^{2}$ and $\tau \sim 2$.

The joint model is fitted using the Extended Quasi Loglikelihood (EQL, Nelder and Pregibon, 1987) maximization. The EQL behaves as a log-likelihood for both mean and dispersion parameters. This justifies an iterative procedure to fit the joint model. First, a GLM is fitted on the mean; then from the estimate of $d$, another GLM is fitted on the dispersion. From the estimate of $\phi$, weights for the next estimate of the GLM on the mean are obtained. This process can be reiterated as many times it is necessary, and allows to entirely fit our joint model (McCullagh and Nelder, 1989).

Statistical tools available in the GLM fitting are also available for each component of the joint model: deviance analysis and Student test. It allows to make some variable selection in order to simplify model expressions. The residuals graphical analysis (which have to be normally distributed) and the q-q plots can be used as indicators of the correctness of the link function for the mean component (Lee and Nelder, 2003). In practice, some evidence can lead to an adequate choice of the link function (McCullagh and Nelder, 1989). For example, a binomial-type explained variable leads to the use of the logit function. However, if a natural choice is not possible and if the identity link function does not provide satisfactory residuals analysis, plotting the adjusted dependent variable versus the linear predictor might help in choosing a more appropriate link function (McCullagh and Nelder, 1989).

In conclusion, all the results obtained on the joint GLM are applicable to the problem of stochastic computer experiments. The novelty proposed in our paper concerns the global sensitivity analysis issue (section 3.2). Moreover, in the following section we extend the joint GLM to the non parametric framework. This kind of model is necessary for the computer experiment outputs which tend to be rather complex and need non parametric modeling.

Remark: A simpler approach consists in building polynomial models for the mean and the variance separately (Vining and Myers, 1990: Bursztyn and Steinberg, 2006). This approach, called dual modeling, consists in repeating calculations with the same sets of controllable variables (which is not necessary in the joint modeling approach). The dual modeling approach has been successfully applied in many situations, especially for robust conception problems. However for our purpose (accurate fitting of the mean and dispersion components), it has been shown that this dual model is less competitive than the joint model (Zabalza et al., 1998; Lee and Nelder, 2003): the dual modeling approach fits the dispersion model given the mean model and this approach does not always lead to optimal fits.

\subsection{Extension to the Generalized Additive Models}

Generalized Additive models (GAM) were introduced by Hastie and Tibshirani (1990) and allow a linear term in the linear predictor $\eta=\sum_{j} \beta_{j} X_{j}$ of equation (1) to be replaced by a sum of smooth functions $\eta=\sum_{j} s_{j}\left(X_{j}\right)$. The $s_{j}($.$) 's are$ unspecified functions that are obtained by fitting a smoother to the data, in an iterative procedure. GAMs provide a flexible method for identifying nonlinear covariate effects in exponential family models and other likelihood-based regression models. The fitting of GAM introduces an extra level 
of iteration in which each spline is fitted in turn assuming the others known. GAM terms can be mixed quite generally with GLM terms in deriving a model.

One common choice for $s_{j}$ is the smoothing spline, i.e. splines with knots at each distinct value of the variables. In regression problems, smoothing splines have to be penalized in order to avoid data overfitting. Wood and Augustin (2002) have described in details how GAMs can be constructed using penalized regression splines. Because numerical models often exhibit strong interactions between input variables, the incorporation of multi-dimensional smooth (for example the bi-dimensional spline term $\left.s_{i j}\left(X_{i}, X_{j}\right)\right)$ is particularly important in our context.

GAMs are generally fitted using penalized likelihood maximization. For this purpose, the likelihood is modified by the addition of a penalty for each smooth function, penalizing its "wiggliness". Namely, the penalized loglikelihood is defined as:

$p \ell=\ell+\sum_{j=1}^{p} \lambda_{j} \int\left(\frac{\partial^{2} s_{j}}{\partial x_{j}^{2}}\right)^{2} d x_{j}$

where $\ell$ is the loglikelihood function, $p$ is the total number of smooth terms and $\lambda_{j}$ are "smoothing parameters" which compromise between goodness of fit and smoothness.

Estimation of these "smoothing parameters" is generally achieved using the GCV score minimization. The GCV score is defined as:

$S_{G C V}=\frac{n d}{(n-D o F)^{2}}$

where $n$ is the number of data, $d$ is the deviance and $D o F$ is the effective degrees of freedom, i.e. the trace of the socalled "hat" matrix. Extension to (E)QL models is straightforward by substituting the likelihood function and the deviance $d$ for their (extended) quasi counterparts. In practice, all the smoothing parameters are jointly updated at each iteration of the fitting procedure of the joint model. To this aim, on every iteration a GLM/GAM is fitted for each trial set of smoothing parameters, and GCV scores are only evaluated at convergence.

We have seen that GAMs extend in a natural way GLMs. Therefore, it would be interesting to extend the joint GLM model to a joint GAM one. Such ideas have been proposed in Rigby and Stasinopoulos (1996) where both the mean and variance were modeled using semi-parametric additive models (Hastie and Tibshirani, 1990). This model is restricted to observations following a Gaussian distribution and is called Mean and Dispersion Additive Model (MADAM). Our model is more general and relaxes the Gaussian assumption as now quasi-distributions are considered: while the MADAM fitting procedure relies on the maximization of the penalized likelihood, the joint GAM maximizes the penalized extended quasi-likelihood. In addition, Rigby and Stasinopoulos (1996) only used cubic regression splines, while our framework allows also the use of multivariate smoothers - e.g. thin plate regression splines. As our model is based on GAMs and by analogy with the denomination "joint GLM", we call it "joint GAM" in the following.

Lastly, it has to be noticed that, within the EQL maximization framework, a large number of models can be considered instead of GAMs. For instance, one can use a GAM for the mean response and a GLM for the dispersion component. In addition, more complex models can also be considered such as Gaussian processes - see Section 2.3

\subsection{Joint modeling with other models}

For some applications, joint GAM could be inadequate, and other models can be proposed. For example, for Gaussian observations, Juutilainen and Röning (2005) have used a neural network model for mean and dispersion. It is shown to be more efficient than joint GLM and joint additive models in a context of numerous explanatory variables (25) and of a large amount of data (100000). They perform an extensive comparison for large data sets between joint neural network model, MADAM, joint local linear regression model and joint linear regression model. While our context of computer experiments is different (we have small data sets), it is interesting to recall their conclusion:

- the neural network joint model gives the best prediction performance;

- MADAM requires a huge amount of memory;

- joint local linear model is extremely time consuming;

- joint linear model is appropriate when simplicity is required.

It is also possible to build a heteroscedastic model based on the Gaussian process ( $\mathrm{Gp}$ ) metamodel (also known as the kriging principle, Sacks et al., 1989). The Gp approach essentially is a kind of linear interpolation built on the property of the multivariate normal distribution. Gp metamodel gives not only a predictor (which is the best linear unbiased predictor) of a computer experiment but also a local indicator of prediction accuracy. For heteroscedastic data, a first approach, proposed by Ginsbourger et al. (2008), consists in modeling the mean of the computer code with a Gp metamodel for which the nugget effect is supposed to vary with the inputs. From this fitted Gp, one can use the estimation of the MSE (given by the Gp model) as the dispersion statistic $d$ introduced in Equation (2). This model does not require any fitting of the dispersion component and we prefer to focus our attention on another method, the joint $\mathrm{Gp}$ model, which is coherent with our previous joint models. Boukouvalas and Cornford (2009) have recently introduced a such joint Gp model for the same purpose. 
The first step of our methodology models the mean by a Gp metamodel (having a nugget effect) estimated on the learning sample. The second step consists in adjusting a second Gp metamodel on the squared residuals. This process can be iterated as in the joint GLM and joint GAM fitting procedure. Due to the presence of a nugget effect in the mean component, the mean Gp is not anymore an exact interpolator and the learning sample residuals can be used for the dispersion model. However, residuals could also be derived from a cross validation method.

\section{Global sensitivity analysis}

\subsection{Deterministic models}

The global SA methods are applicable to deterministic computer code, e.g. for which the same set of input variables always leads to the same response value. This is considered by the following model:

$$
\begin{aligned}
f: \mathbb{R}^{p} & \rightarrow \mathbb{R} \\
\mathbf{X} & \mapsto Y=f(\mathbf{X})
\end{aligned}
$$

where $f(\cdot)$ is the model function (possibly analytically unknown $), \mathbf{X}=\left(X_{1}, \ldots, X_{p}\right)$ are $p$ independent inputs and $Y$ is the output. In our problem, $\mathbf{X}$ is uncertain and considered as a random vector with known distribution which reflects this uncertainty. Therefore, $Y$ is also a random variable, whose distribution is unknown. In this section, let us recall some basic ideas on the variance-based sensitivity indices, called Sobol indices, applied on this model.

Among quantitative methods, variance-based methods are the most often used (Saltelli et al., 2000). The main idea of these methods is to evaluate how the variance of an input or a group of inputs contributes into the variance of output. We start from the following variance decomposition:

$\operatorname{Var}[Y]=\operatorname{Var}\left[\mathbb{E}\left(Y \mid X_{i}\right)\right]+\mathbb{E}\left[\operatorname{Var}\left(Y \mid X_{i}\right)\right]$,

which is known as the total variance theorem. The first term of this equality, named variance of the conditional expectation, is a natural indicator of the importance of $X_{i}$ into the variance of $Y$ : the greater the importance of $X_{i}$, the greater is $\operatorname{Var}\left[\mathbb{E}\left(Y \mid X_{i}\right)\right]$. Most often, this term is divided by $\operatorname{Var}[Y]$ to obtain a sensitivity index in $[0,1]$.

To express the sensitivity indices, we use the unique decomposition of any integrable function on $[0,1]^{p}$ into a sum of elementary functions (see for example Sobol, 1993):

$$
\begin{array}{r}
f\left(X_{1}, \cdots, X_{p}\right)=f_{0} \\
+\sum_{i}^{p} f_{i}\left(X_{i}\right)+\sum_{i<j}^{p} f_{i j}\left(X_{i}, X_{j}\right) \\
+\ldots+f_{12 . . p}\left(X_{1}, \cdots, X_{p}\right),
\end{array}
$$

where $f_{0}$ is a constant and the other functions verify the following conditions:

$\int_{0}^{1} f_{i_{1}, \ldots, i_{s}}\left(x_{i_{1}}, \ldots, x_{i_{s}}\right) d x_{i_{k}}=0$ $\forall k=1, \ldots, s, \forall\left\{i_{1}, \ldots, i_{s}\right\} \subseteq\{1, \ldots, p\}$. If the $X_{i}$ s are mutually independent, the decomposition (7) is valid for any distribution functions for the $X_{i} \mathrm{~s}$.

From (7), the following decomposition of the model output variance is possible (Sobol, 1993):

$$
\begin{array}{r}
\operatorname{Var}[Y]=\sum_{i}^{p} V_{i}(Y)+\sum_{i<j}^{p} V_{i j}(Y)+\sum_{i<j<k}^{p} V_{i j k}(Y) \\
+\ldots+V_{12 . . p}(Y)
\end{array}
$$

where $V_{i}(Y)=\operatorname{Var}\left[\mathbb{E}\left(Y \mid X_{i}\right)\right], V_{i j}(Y)=\operatorname{Var}\left[\mathbb{E}\left(Y \mid X_{i} X_{j}\right)\right]-V_{i}(Y)-$ $V_{j}(Y), \ldots$ One can thus define the sensitivity indices by:

$$
\begin{aligned}
& S_{i}=\frac{\operatorname{Var}\left[\mathbb{E}\left(Y \mid X_{i}\right)\right]}{\operatorname{Var}(Y)}=\frac{V_{i}(Y)}{\operatorname{Var}(Y)}, \\
& \quad S_{i j}=\frac{V_{i j}(Y)}{\operatorname{Var}(Y)}, \quad S_{i j k}=\frac{V_{i j k}(Y)}{\operatorname{Var}(Y)}, \ldots
\end{aligned}
$$

These coefficients are called the Sobol indices, and can be used for any complex model functions $f$. The second order index $S_{i j}$ expresses sensitivity of the model to the interaction between the variables $X_{i}$ and $X_{j}$ (without the first order effects of $X_{i}$ and $X_{j}$ ), and so on for higher orders effects. The interpretation of these indices is natural as their sum is equal to one (thanks to equation (9)): the larger and close to one an index value, the greater is the importance of the variable or the group of variables linked to this index.

For a model with $p$ inputs, the number of Sobol indices is $2^{p}-1$; leading to an intractable number of indices as $p$ increases. Thus, to express the overall sensitivity of the output to an input $X_{i}$, Homma and Saltelli (1996) introduce the total sensitivity index:

$S_{T_{i}}=S_{i}+\sum_{j \neq i} S_{i j}+\sum_{j \neq i, k \neq i, j<k} S_{i j k}+\ldots=\sum_{l \in \# i} S_{l}$

where \# $i$ represents all the "non-ordered" subsets of indices containing index $i$. Thus, $\sum_{l \in \# i} S_{l}$ is the sum of all the sensitivity indices containing $i$ in their index. For example, for a model with three input variables, $S_{T_{1}}=S_{1}+S_{12}+S_{13}+S_{123}$.

The estimation of these indices can be done by MonteCarlo simulations or by alternative methods (Saltelli et al., 2000). Recent algorithms have also been introduced to reduce the number of required model evaluations significantly. As explained in the introduction, a powerful method consists in replacing complex computer models by metamodels which have negligible calculation time (e.g. Volkova et al., 2008). Estimation of Sobol indices by Monte-Carlo techniques with their confidence intervals (requiring thousand of simulations) can then be done using these response surfaces.

\subsection{Stochastic models}

In this work, models containing some intrinsic alea, which is described as an uncontrollable random input variable $\varepsilon$, are 
called "stochastic computer models". Let us recall the example proposed in the introduction where $\varepsilon$ is a random field whose each realization is governed by a random seed value. We consider the random field $\varepsilon$ as an uncontrollable variable when this random field is too complex to be described or synthesized by a reasonable number of scalar parameters.

In the following, the expectation and variance operators involve averaging over the distribution of $(\mathbf{X}, \varepsilon)$, unless another distribution is indicated. Similarly from equation (5), consider the following (stochastic) model:

$$
\begin{aligned}
g: \mathbb{R}^{p} & \rightarrow \mathbb{R} \\
\mathbf{X} & \mapsto Y=f(\mathbf{X})+v(\varepsilon, \mathbf{X}: \varepsilon)
\end{aligned}
$$

where $\mathbf{X}$ are the $p$ controllable input variables (independent random variables), $Y$ is the output, $f(\cdot)$ is the deterministic part of the model function and $v(\cdot)$ is the stochastic part of the model function. Let $\mathbb{E}_{\mathcal{E}}(v)=0$ which means that $v(\cdot)$ is centered relatively to $\varepsilon$ : we put inside $f(\cdot)$ a possible constant term involved by $v(\cdot)$. The notation $v(\varepsilon, \mathbf{X}: \varepsilon)$ means that $v$ depends only on $\varepsilon$ and on the interactions between $\varepsilon$ and $\mathbf{X}$. The additive form of equation (12) is deduced directly from the decomposition of the function $g$ into a sum of elementary functions depending on $(\mathbf{X}, \varepsilon)$ (like the decomposition in Eq. (7)).

For a stochastic model (12), the joint models introduced in section 2 enables us to recover two GLMs, two GAMs or two Gps:

$Y_{m}(\mathbf{X})=\mathbb{E}(Y \mid \mathbf{X})=\mathbb{E}_{\mathcal{\varepsilon}}(Y \mid \mathbf{X})$

by the mean component (Eq. (1)), and

$Y_{d}(\mathbf{X})=\operatorname{Var}(Y \mid \mathbf{X})=\operatorname{Var}_{\varepsilon}(Y \mid \mathbf{X})$

by the dispersion component (Eq. (2)). If there is no uncontrollable variable $\varepsilon$, it leads to a deterministic model case with $Y_{d}(\mathbf{X})=\operatorname{Var}(Y \mid \mathbf{X})=0$. By using the total variance theorem (Eq. (6)), the variance of the output variable $Y$ can be decomposed by:

$$
\begin{aligned}
\operatorname{Var}(Y) & =\operatorname{Var}_{\mathbf{X}}[\mathbb{E}(Y \mid \mathbf{X})]+\mathbb{E}_{\mathbf{X}}[\operatorname{Var}(Y \mid \mathbf{X})] \\
& =\operatorname{Var}_{\mathbf{X}}\left[Y_{m}(\mathbf{X})\right]+\mathbb{E}_{\mathbf{X}}\left[Y_{d}(\mathbf{X})\right]
\end{aligned}
$$

According to model (12), $Y_{m}(\mathbf{X})$ is the deterministic model part, and $Y_{d}(\mathbf{X})$ is the variance of the stochastic model part:

$Y_{m}(\mathbf{X})=f(\mathbf{X})$

$Y_{d}(\mathbf{X})=\operatorname{Var}_{\varepsilon}[v(\varepsilon, \mathbf{X}: \varepsilon) \mid \mathbf{X}]$.

The variances of $Y$ and $Y_{m}(\mathbf{X})$ are now decomposed according to the contributions of their input variables $\mathbf{X}$. For $Y$, the same decomposition than for deterministic models holds (Eq. (9)). However, it includes the additional term $\mathbb{E}_{\mathbf{X}}\left[Y_{d}(\mathbf{X})\right]$ (the mean of the dispersion component) deduced from equation (15). Consequently,

$$
\begin{array}{r}
\operatorname{Var}(Y)=\sum_{i}^{p} V_{i}(Y)+\sum_{i<j}^{p} V_{i j}(Y)+\sum_{i<j<k}^{p} V_{i j k}(Y) \\
+\ldots+V_{12 . . p}(Y)+\mathbb{E}_{\mathbf{X}}\left[Y_{d}(\mathbf{X})\right]
\end{array}
$$

where $V_{i}(Y)=\operatorname{Var}_{X_{i}}\left[\mathbb{E}\left(Y \mid X_{i}\right)\right], V_{i j}(Y)=\operatorname{Var}_{\left(X_{i}, X_{j}\right)}\left[\mathbb{E}\left(Y \mid X_{i} X_{j}\right)\right]-$ $V_{i}(Y)-V_{j}(Y), \ldots$ For the mean component $Y_{m}(\mathbf{X})$ that we note $Y_{m}$ for easing the notation, we have

$$
\begin{array}{r}
\operatorname{Var}\left(Y_{m}\right)=\sum_{i}^{p} V_{i}\left(Y_{m}\right)+\sum_{i<j}^{p} V_{i j}\left(Y_{m}\right)+\sum_{i<j<k}^{p} V_{i j k}\left(Y_{m}\right) \\
+\ldots+V_{12 . . p}\left(Y_{m}\right)
\end{array}
$$

By noticing that

$$
\begin{aligned}
V_{i}\left(Y_{m}\right) & =\operatorname{Var}_{X_{i}}\left[\mathbb{E}_{\mathbf{X}}\left(Y_{m} \mid X_{i}\right)\right]=\operatorname{Var}_{X_{i}}\left\{\mathbb{E}_{\mathbf{X}}\left[\mathbb{E}_{\mathcal{\varepsilon}}(Y \mid \mathbf{X}) \mid X_{i}\right]\right\} \\
& =\operatorname{Var}_{X_{i}}\left[\mathbb{E}_{\mathbf{X}, \varepsilon}\left(Y \mid X_{i}\right)\right]=V_{i}(Y)
\end{aligned}
$$

and from equation (10), the sensitivity indices for the variable $Y$ according to the controllable variables $\mathbf{X}=\left(X_{i}\right)_{i=1 \ldots p}$ can be computed using:

$S_{i}=\frac{V_{i}\left(Y_{m}\right)}{\operatorname{Var}(Y)}, \quad S_{i j}=\frac{V_{i j}\left(Y_{m}\right)}{\operatorname{Var}(Y)}, \quad \ldots$

These Sobol indices can be computed by classical MonteCarlo techniques, the same ones used in the deterministic model case. These algorithms are applied on the metamodel defined by the mean component $Y_{m}$ of the joint model.

Thus, all terms contained in $\operatorname{Var}_{\mathbf{X}}\left[Y_{m}(\mathbf{X})\right]$ of the equation (15) have been considered. It remains to estimate $\mathbb{E}_{\mathbf{X}}\left[Y_{d}(\mathbf{X})\right]$ by a simple numerical integration of $Y_{d}(\mathbf{X})$ following the distribution of $\mathbf{X} . Y_{d}(\mathbf{X})$ is evaluated with a metamodel, for example the dispersion component of the joint model. $\mathbb{E}_{\mathbf{X}}\left[Y_{m}(\mathbf{X})\right]$ includes all the decomposition terms of $\operatorname{Var}(Y)$ (according to $\mathbf{X}$ and $\varepsilon$ ) not taken into account in $\operatorname{Var}_{\mathbf{X}}\left[Y_{m}(\mathbf{X})\right]$ i.e. all terms involving $\varepsilon$. Therefore, the total sensitivity index of $\varepsilon$ is

$S_{T_{\varepsilon}}=\frac{\mathbb{E}_{\mathbf{X}}\left[Y_{m}(\mathbf{X})\right]}{\operatorname{Var}(Y)}$.

As $Y_{d}(\mathbf{X})$ is a positive random variable, positivity of $S_{T_{\varepsilon}}$ is guaranteed. In practice, $\operatorname{Var}(Y)$ can be estimated from the data or from simulations of the fitted joint model:

$\operatorname{Var}(Y)=\operatorname{Var}_{\mathbf{X}}\left[Y_{m}(\mathbf{X})\right]+\mathbb{E}_{\mathbf{X}}\left[Y_{m}(\mathbf{X})\right]$

If $\operatorname{Var}(Y)$ is computed from the data, it seems preferable to estimate $\mathbb{E}_{\mathbf{X}}\left[Y_{m}(\mathbf{X})\right]$ with $\operatorname{Var}(Y)-\operatorname{Var}_{\mathbf{X}}\left[Y_{m}(\mathbf{X})\right]$ to satisfy equation (15). In our applications, the total variance will be estimated using the fitted joint model (Eq. (22)). 
Finally, let us note that we cannot quantitatively distinguish the various contributions in $S_{T_{\varepsilon}}\left(S_{\mathcal{\varepsilon}}, S_{i \varepsilon}, S_{i j \varepsilon}, \ldots\right)$. Indeed, it is not possible to combine the functional anova decomposition of $Y_{m}(\mathbf{X})$ with the functional anova decomposition of $Y_{d}(\mathbf{X})$ in order to deduce the unknown sensitivity indices. Finding a way to form some composite indices still remains an open problem which needs further research. However, we argue that the analysis of the terms in the regression model $Y_{d}$ and their $t$-values give useful qualitative information. For example, if an input variable $X_{i}$ is not present in $Y_{d}$, we can deduce the following correct information: $S_{i \varepsilon}=0$. Moreover, if the $t$-values analysis and the deviance analysis show that an input variable $X_{i}$ has a smaller influence than another input variable $X_{j}$, we can suppose that the interaction between $X_{i}$ and $\varepsilon$ is less influential than the interaction between $X_{j}$ and $\varepsilon$.

In conclusion, this new approach, based on joint models to compute Sobol sensitivity indices, is useful if the following conditions hold:

- if the computer model contains some uncontrollable variables (the model is no more deterministic but stochastic);

- if a metamodel is needed due to large CPU times of the computer model;

- if some of the uncontrollable variables interact with some controllable input ones;

- if some information about the influence of the interactions between the uncontrollable variables and the other input variables is of interest.

\section{Applications}

\subsection{An analytic test case: the Ishigami function}

The proposed method is first illustrated on an artificial analytical model with 3 input variables, called the Ishigami function (Homma and Saltelli, 1996; Saltelli et al., 2000):

$Y=f\left(X_{1}, X_{2}, X_{3}\right)=\sin \left(X_{1}\right)+7 \sin \left(X_{2}\right)^{2}+0.1 X_{3}^{4} \sin \left(X_{1}\right)$

where $X_{i} \sim \mathscr{U}[-\pi ; \pi]$ for $i=1,2,3$. For this function, all the Sobol sensitivity indices $\left(S_{1}, S_{2}, S_{3}, S_{12}, S_{13}, S_{23}, S_{123}, S_{T_{1}}\right.$, $\left.S_{T_{2}}, S_{T_{3}}\right)$ are known. This function is used in most intercomparison studies of global sensitivity analysis algorithms. In our study, the classical problem is altered by considering $X_{1}$ and $X_{2}$ as the controllable input random variables, and $X_{3}$ as an uncontrollable input random variable. It means that the $X_{3}$ random values are not used in the modeling procedure; this variable is considered to be inaccessible. However, sensitivity indices have the same theoretical values as in the standard case.
For this analytical function case, it is easy to obtain the exact mean and dispersion models by deriving (via analytical integration) the analytical expressions of the mean component $Y_{m}\left(X_{1}, X_{2}\right)$ and dispersion component $Y_{d}\left(X_{1}, X_{2}\right)$ :

$$
\begin{aligned}
Y_{m}\left(X_{1}, X_{2}\right) & =\mathbb{E}\left(Y \mid X_{1}, X_{2}\right) \\
& =\left(1+\frac{\pi^{4}}{50}\right) \sin \left(X_{1}\right)+7\left[\sin \left(X_{2}\right)\right]^{2}, \\
Y_{d}\left(X_{1}, X_{2}\right) & =\operatorname{Var}\left(Y \mid X_{1}, X_{2}\right) \\
& =\pi^{8}\left(\frac{1}{900}-\frac{1}{2500}\right)\left[\sin \left(X_{1}\right)\right]^{2}=Y_{d}\left(X_{1}\right) .
\end{aligned}
$$

\subsubsection{Metamodeling}

For the model fitting, 1000 Monte Carlo samples of $\left(X_{1}, X_{2}, X_{3}\right)$ were simulated leading to 1000 observations for $Y$. No replication is made in the $\left(X_{1}, X_{2}\right)$ plane because it has been shown that repeating calculations with the same sets of controllable variables is inefficient in the joint modeling approach (Zabalza et al., 1998; Lee and Nelder, 2003). Therefore, we argue that it is better to keep all the possible experiments to optimally cover the input variable space (which can be highly dimensional in real problems). In practice, quasiMonte Carlo sequences will be preferred to pure Monte Carlo samples (Fang et al., 2006).

In this section, the GLM, GAM and Gp model (with their relative joint extensions) are compared (see Table 1). To compare the predictivity of different metamodels, we use the predictivity coefficient $Q_{2}$, which is the determination coefficient $R^{2}$ computed from a test sample (composed here by 10000 randomly chosen points). For each joint model, $Q_{2}$ is computed on the mean component.

The simple GLM is a fourth order polynomial. Only the explanatory terms are selected in our regression model using analysis of deviance and the Fisher statistics. The Student test on the regression coefficients and residuals graphical analysis make it possible to judge the goodness of fit. We see that it remains $39 \%$ of non explained deviance due to the model inadequacy and/or to the uncontrollable variable. The mean component of the joint GLM gives the same model as the simple GLM. For the dispersion component, using analysis of deviance techniques, no significant explanatory variable was found. Thus, the dispersion component is supposed to be constant; and the joint GLM is equivalent to the simple GLM approach - but with a different fitting process.

Studying now the non parametric modeling, we start by the simple GAM fitting where we have kept some parametric terms by applying a term selection procedure. The predictivity coefficient of the mean component of the joint GAM is slightly better than the predictivity coefficient of the simple GAM. However, the explained deviance given by the joint GAM mean component is clearly larger than the one given 
Table 1 Results for the fitting of different metamodels for the Ishigami function. $D_{\text {expl }}$ (the explained deviance of the model) and $Q_{2}$ (the predictivity coefficient of the model) are expressed in percent. For the joint models, $D_{\text {expl }}$ and $Q_{2}$ are those of the mean component $Y_{m}$. In the formulas for GAM, $s_{1}(\cdot), s_{2}(\cdot)$ and $s_{d 1}(\cdot)$ are three spline terms.

\begin{tabular}{cccc}
\hline & $D_{\text {expl }}$ & $Q_{2}$ & Formula \\
\hline Simple GLM & 61.3 & 60.8 & $Y=1.92+2.69 X_{1}+2.17 X_{2}^{2}-0.29 X_{1}^{3}-0.29 X_{2}^{4}$ \\
Joint GLM & 61.3 & 60.8 & $Y_{m}=1.92+2.69 X_{1}+2.17 X_{2}^{2}-0.29 X_{1}^{3}-0.29 X_{2}^{4}$ \\
& & & $\log \left(Y_{d}\right)=1.73$ \\
Simple GAM & 76.8 & 75.1 & $Y=3.76-2.67 X_{1}+s_{1}\left(X_{1}\right)+s_{2}\left(X_{2}\right)$ \\
Joint GAM & 92.8 & 75.5 & $Y_{m}=3.75-3.06 X_{1}+s_{1}\left(X_{1}\right)+s_{2}\left(X_{2}\right)$ \\
& & & $\log \left(Y_{d}\right)=0.59+s_{d 1}\left(X_{1}\right)$ \\
Simple Gp & - & 75.0 & - \\
Joint Gp & - & 75.0 & - \\
\hline
\end{tabular}

by the simple GAM approach. Even if this could be related to an increasing number of parameters, as the number of parameters remains very small compared to the data size (1000), it is certainly explained by the fact that GAMs are more flexible than GLMs. This demonstrates the efficiency of the joint modeling of the mean and dispersion when heteroscedasticity is involved. Indeed, the joint procedure leads to appropriate prior weights for the mean component. The joint GAM improves both the joint GLM and simple GAM approaches:

(a) due to the GAMs flexibility, the explanatory variable $X_{1}$ is identified to model the dispersion component (the interaction between $X_{1}$ and the uncontrollable variable $X_{3}$ is therefore retrieved);

(b) the joint GAM explained deviance (93\%) for the mean component is clearly larger than the simple GAM and joint GLM ones (joint GLM: 61\%, simple GAM: 77\%).

For the Gp metamodel fitting, we use the methodology of Marrel et al. (2008) which include in the model a linear regression part and a Gp defined by a generalized exponential covariance. We obtain for the simple Gp the predictivity coefficient $Q_{2}=75.0 \%$, which is extremely close to the one of the simple GAM $\left(Q_{2}=75.1 \%\right)$. The variance of the nugget effect (additional error with constant variance) introduced in the Gp model is estimated to $25.9 \%$ of the total variance, which is close to the expected value $\left(1-Q_{2}=25.0 \%\right)$. We can also fit, at present, a Gp model on the squared residuals to obtain a joint Gp model (cf. section 2.3. In order to understand which inputs act in the dispersion component, we compute the Sobol sensitivity indices of the dispersion component using a Monte Carlo algorithm: $S_{Y_{d}}\left(X_{1}\right)=0.996$ and $S_{Y_{d}}\left(X_{2}\right)=0.001$. These results draw the same conclusion than those obtained from the dispersion component equation of the joint GAM: $X_{2}$ is not an explanatory factor for the dispersion. This also leads to the right conclusion that only $X_{1}$ interacts with the uncontrollable variable $X_{3}$ in the Ishigami function (23).

Let us now perform some graphical analyses in order to compare the results for the three joint models Joint GLM, joint GAM and Joint Gp. Figure 1 shows the observed re- sponse against the predicted values for the three models. First, the advantage of the GAM and Gp approaches are visible in the Figure 1 as the dispersion around the $y=x$ line is clearly reduced compared to the joint GLM dispersion. Graphical comparisons between Joint GAM and Joint Gp results do not provide any advantage for one particular model: similar biases are shown. Second, using the GAM model, Figure 2 compares the obtained residuals of a non parametric simple model (homoscedastic) with the obtained residuals of a non parametric joint model (heteroscedastic). The deviance residuals for the mean component of the joint GAM seem to be more homogeneously dispersed around the $x$-axis; leading to a better prediction on the whole range of the observations. Thus, the joint approach is more competitive than the simple one. From this simple graphical analyses, we conclude that a non parametric joint model (GAM or $\mathrm{Gp}$ ) has to be preferred to other models (simple and/or parametric).

In order to make a finer comparison between GLM, GAM and $\mathrm{Gp}$ models, we examine how well they predict the mean $Y_{m}\left(X_{1}, X_{2}\right)$ at inputs for which we have no data. We can also compare the different dispersion models $Y_{d}\left(X_{1}\right)$. The exact analytical expressions of $Y_{m}$ and $Y_{d}$ are given in Eq. (24). Let us remark that we visualize $Y_{d}$ versus $X_{1}$ only because, for GLM and GAM dispersion models, there is no dependence in $X_{2}$ and, for the Gp dispersion model, there is an extremely small $X_{2}$-dependence (we then take $X_{2}=0$ ). Figure 3 plots the theoretical $Y_{m}$ and $Y_{d}$ surfaces (left panels) and their estimates derived from the fitted joint GLM, joint GAM and Joint Gp models. As shown before, the joint GLM is irrelevant for the mean component and for the dispersion component. The joint GAM fully reproduces the mean component, while joint $\mathrm{Gp}$ gives a rather good approximation, but with small noise. Indeed, spline terms of GAM are perfect smoothers while Gp predictor is impacted by residual noise on the observations: the nugget effect does not allow to suppress all the noise induced by the uncontrollable variable. For the dispersion component, joint GP and joint GAM give result of the same quality: these models correctly reproduce the overall behaviour but with small inadequacies, 

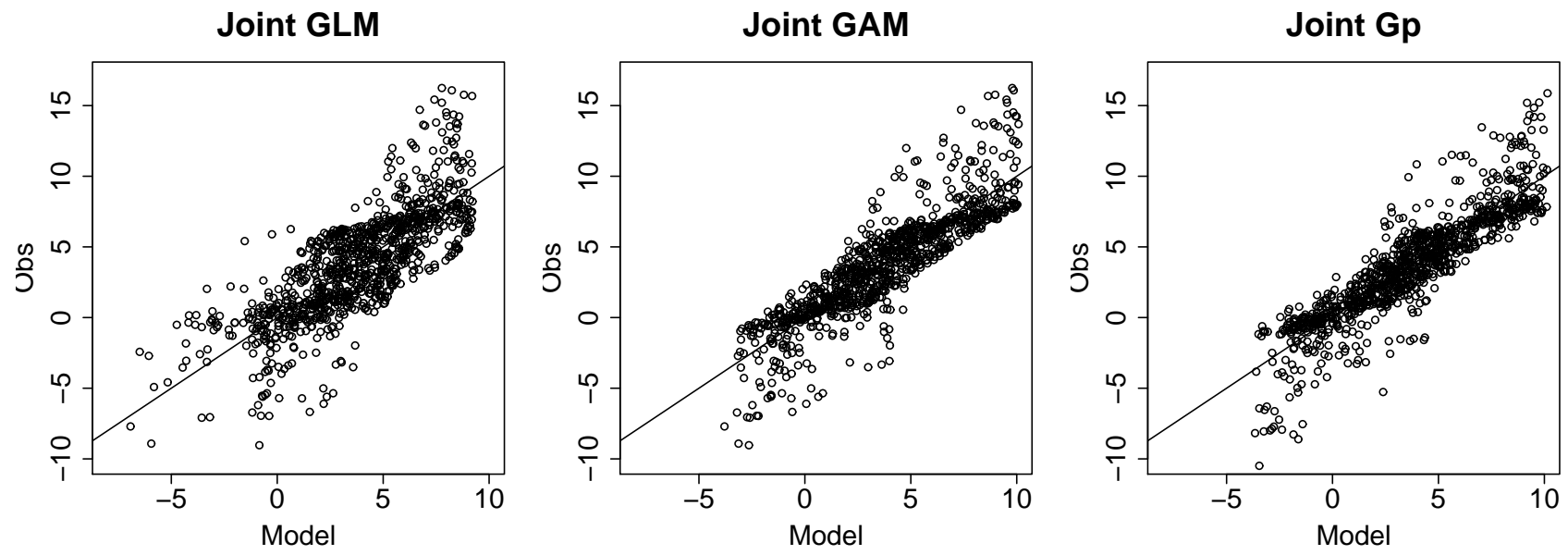

Fig. 1 Observed response variable versus the predicted values for the three joint models: Joint GLM, Joint GAM and joint Gp (Ishigami application).
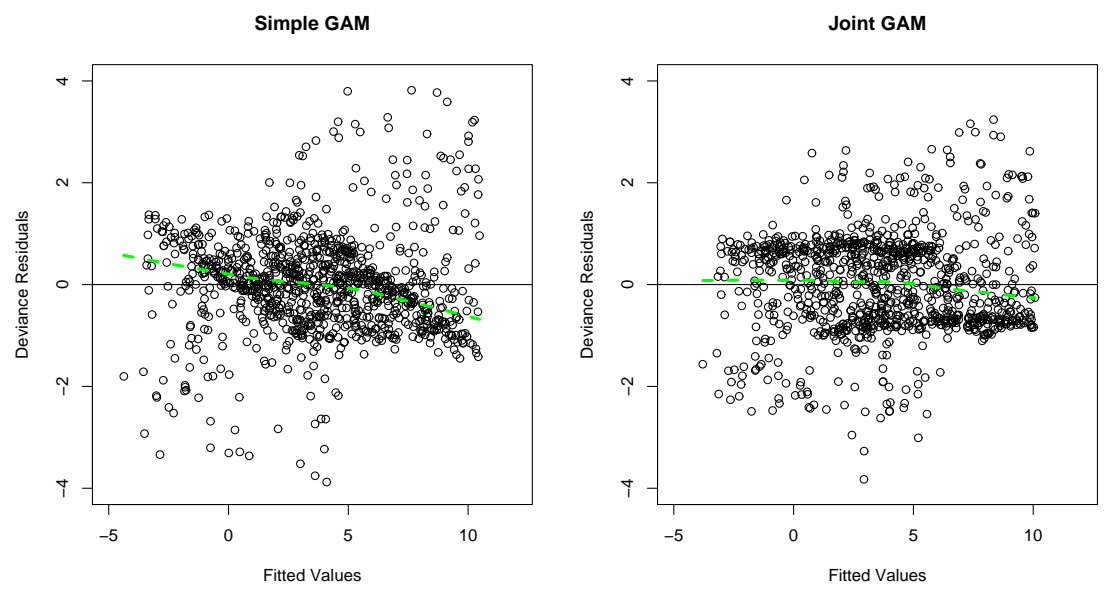

Fig. 2 Deviance residuals for the simple and joint GAMs versus the fitted values (Ishigami application). Dashed lines correspond to local polynomial smoothers.

probably caused by overfitting problems. For the two dispersion models, fitted observations have been taken from the residuals of the mean component learning sample. It would be convenient, in a future work, to test another solution by taking predicted residuals, for example by applying a cross validation procedure.

We conclude that the joint GAM and joint Gp both adequately model the stochastic analytical model (the Ishigami function (23)). We let some fine comparisons between joint GAM and joint $\mathrm{Gp}$ for another study including a relevant analytical application. For example, an analytical model with strong and high order interactions will probably show the superiority of the Gp joint model (because spline high or- der interaction terms are difficult to include inside a GAM). Therefore, in the industrial application of section 4.2, we only use the models based on GLM and GAM, while Gp could also be applied.

\subsubsection{Sobol indices}

Table 2 depicts the Sobol sensitivity indices for the joint GLM, the joint GAM and joint Gp using equations (20) and (21). The standard deviation estimates $(s d)$ are obtained from 100 repetitions of the Monte-Carlo estimation procedure (which uses $10^{4}$ model computations for one index estimation). When this Monte-Carlo procedure is used to es- 

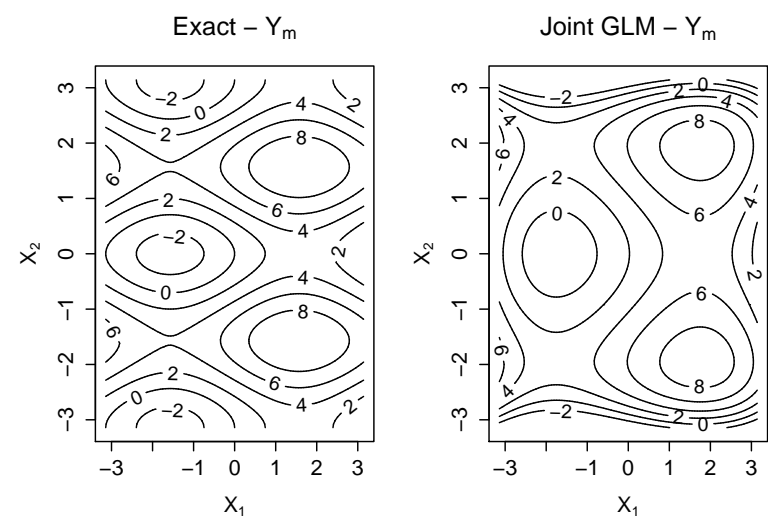

Exact $-Y_{d}$

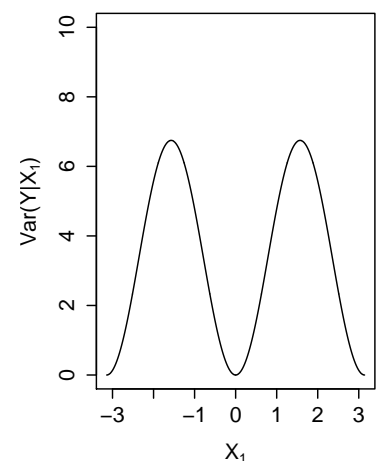

Joint GLM - Y

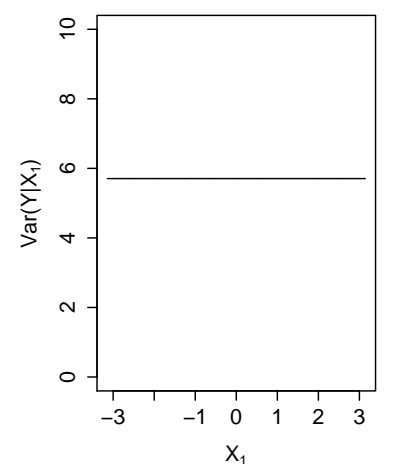

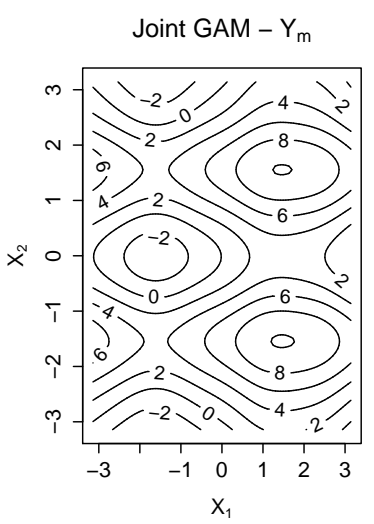

Joint GAM $-Y_{d}$

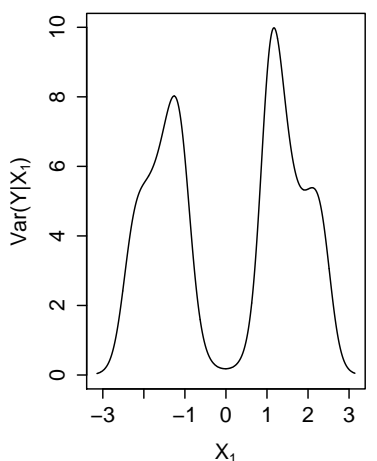

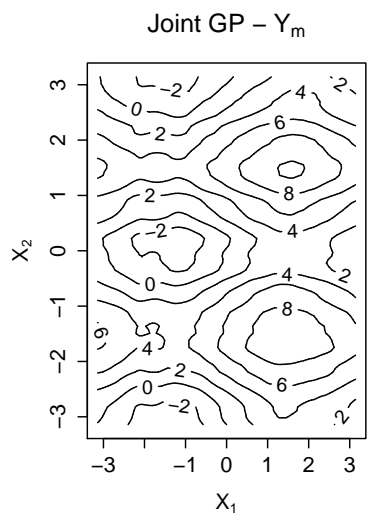

Joint GP $-Y_{d}$

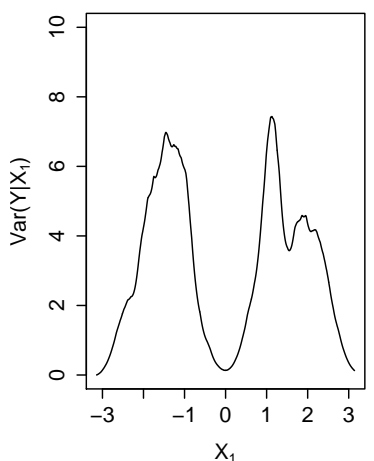

Fig. 3 Mean component (up) and dispersion component (down) for the exact analytical model, Joint GLM, Joint GAM and Joint Gp (Ishigami application).

timate the Sobol index, we report "MC" in the "Method" column; while "Eq" (resp. " $S_{Y_{d}}$ ") indicates that the sensitivity indices have been deduced from the joint model regressive equations (resp. from the sensitivity analysis of the dispersion $\left.Y_{d}\right)$. Therefore, no estimation errors $(s d)$ are associated to these indices (except for total indices $S_{T_{i}}$ which can be deduced from $S_{i}$ ). When no quantitative deduction on the sensitivity index can be made with this process, we have put a variation interval which borders the true value. These variation intervals are deduced from the elementary relations between sensitivity indices (e.g. $S_{1} \leq S_{T_{1}}, S_{13} \leq S_{T_{3}}$, etc).

The joint GLM gives only a good estimation of $S_{1}$, while $S_{2}$ and $S_{T_{3}}$ are badly estimated (errors greater than $30 \%$ ). $S_{12}$ is correctly estimated to zero by looking directly at the joint GLM mean component formula (see Table 1). However, some conclusions drawn from the GLM dispersion component formula (which is a constant) are wrong. As no explanatory variable is involved in this formula, the deduced interaction indices are equal to zero: $S_{13}=S_{23}=S_{123}=0$. Thus, $S_{3}=S_{T_{3}}=0.366$ while the correct values of $S_{3}$ and $S_{T_{3}}$ are respectively zero and 0.243 .

Contrary to the joint GLM, the joint GAM and joint Gp give good approximations of all the Sobol indices. Their largest errors concern $S_{T_{3}}$ for the joint GAM (7\%-error) and joint $\mathrm{Gp}$ (16\%-error). Moreover, the deductions drawn from the model formulas (see Table 1 ) are correct $\left(S_{T_{2}}=S_{2}, S_{12}=\right.$ $S_{23}=S_{123}=0$ ). The only drawback of this joint modelbased method is that some indices remain unknown due to the non separability of the dispersion component effects. However, it can be deduced that $S_{13}$ is non null due to the explicative effect of $X_{1}$ in the dispersion component. The deduced interval variations provide also useful information concerning the potential influence of the interactions.

Table 3 gives the Sobol indices computed by the same Monte-Carlo procedure using two classical metamodels as the simple GAM and the simple Gp. To estimate the first order Sobol indices $S_{i}=V_{i}\left(Y_{m}\right) / \operatorname{Var}(Y)$ (for $i=1,2$ ), the metamodel is used to compute $V_{i}\left(Y_{m}\right)$ and the observed data (the 1000 observations of $Y$ ) to compute $\operatorname{Var}(Y)$. To estimate the total sensitivity index $S_{T_{3}}$ of the uncontrollable variable, the metamodel predictivity coefficient $Q_{2}$ is used. In fact, by supposing that the metamodels fit correctly the computer code, one deduces that all the unexplained part of these metamodels is due to the uncontrollable variable: $S_{T_{3}}=1-Q_{2}$. This is a strong hypothesis, which is verified here due to the simplicity of the analytical function. However, it will not be satisfied for all application cases: in practical and complex situations, the $Q_{2}$ estimation (usually done by a crossvalidation method) can be difficult and subject to caution. For the Ishigami function, $S_{1}, S_{2}, S_{T_{3}}$ are correctly estimated. 
Table 2 Sobol sensitivity indices (with standard deviations) for the Ishigami function: exact and estimated values from joint GLM and joint GAM. "Method" indicates the estimation method: MC for the Monte-Carlo procedure, Eq for a deduction from the model equations and $S_{Y_{d}}$ for a deduction from the sensitivity analysis of $Y_{d}(\mathbf{X})$.

\begin{tabular}{|c|c|c|c|c|c|c|c|c|c|c|}
\hline \multirow{2}{*}{ Indices } & \multirow{2}{*}{$\begin{array}{l}\text { Exact } \\
\text { Values }\end{array}$} & \multicolumn{3}{|c|}{ Joint GLM } & \multicolumn{3}{|c|}{ Joint GAM } & \multicolumn{3}{|c|}{ Joint Gp } \\
\hline & & Values & $s d$ & Method & Values & $s d$ & Method & Values & $s d$ & $\overline{\text { Method }}$ \\
\hline$S_{1}$ & 0.314 & 0.314 & $4 \mathrm{e}-3$ & MC & 0.325 & $5 e-3$ & $\mathrm{MC}$ & 0.292 & $7 \mathrm{e}-3$ & MC \\
\hline$S_{2}$ & 0.442 & 0.318 & $5 e-3$ & MC & 0.414 & $5 e-3$ & MC & 0.417 & $7 e-3$ & MC \\
\hline$S_{T_{3}}$ & 0.244 & 0.366 & $2 \mathrm{e}-3$ & MC & 0.261 & $2 e-3$ & MC & 0.205 & $1 \mathrm{e}-3$ & MC \\
\hline$S_{12}$ & 0 & 0 & - & $\mathrm{Eq}$ & 0 & - & $\mathrm{Eq}$ & 0.004 & $7 e-3$ & MC \\
\hline$S_{13}$ & 0.244 & 0 & - & $\mathrm{Eq}$ & ] $0,0.261]$ & - & $\mathrm{Eq}$ & ] $0,0.205]$ & - & $S_{Y_{d}}$ \\
\hline$S_{23}$ & 0 & 0 & - & $\mathrm{Eq}$ & 0 & - & $\mathrm{Eq}$ & 0 & - & $S_{Y_{d}}$ \\
\hline$S_{123}$ & 0 & 0 & - & $\mathrm{Eq}$ & 0 & - & $\mathrm{Eq}$ & 0 & - & $S_{Y_{d}}^{a}$ \\
\hline$S_{T_{1}}$ & 0.557 & 0.314 & $4 e-3$ & $\mathrm{Eq}$ & ] $0.325,0.586]$ & - & $\mathrm{Eq}$ & ] $0.292,0.497]$ & - & $S_{Y_{d}}$ \\
\hline$S_{T_{2}}$ & 0.443 & 0.318 & $5 e-3$ & $\mathrm{Eq}$ & 0.414 & $5 e-3$ & $\mathrm{Eq}$ & 0.417 & $7 e-3$ & $S_{Y_{d}}$ \\
\hline$S_{3}$ & 0 & 0.366 & $2 e-3$ & $\mathrm{Eq}$ & {$[0,0.261]$} & - & $\mathrm{Eq}$ & {$[0,0.205]$} & - & $S_{Y_{d}}^{d}$ \\
\hline
\end{tabular}

$S_{12}$ can be deduced from the formula for the simple GAM (see Table 1) and estimated by Monte-Carlo method for the Gp model. However, any other sensitivity indices can be proposed as no dispersion modeling is involved.

Remark: Estimating the nugget effect variance of the $G p$ model mean component gives another estimation of the total sensitivity index of the uncotrollable variable. In this example, the variance of the nugget effect has been estimated to $25.9 \%$ of the total variance, which is close to the exact value (24.4\%). However, this estimation can be difficult in more complex situations, because of a difficult optimization step while fitting the Gp model (Fang et al., 2006; Marrel et al., 2008).

In conclusion, this example shows that the joint non parametric models can adjust complex heteroscedastic situations for which classical metamodels are inadequate. Moreover, the joint models offer a theoretical basis to compute efficiently global sensitivity indices of stochastic models.

\subsubsection{Convergence study}

In order to provide some practical guidance for the sampling size issue, we perform a convergence study for the estimation of the joint GAM and the associated sensitivity indices. Figure 4 shows some convergence results for a learning sample size $n$ varying between 30 to 200 by step of 5 . The learning points are sampled by the simple Monte Carlo technique. The predictivity coefficient $Q_{2}$ is obtained from a test sample (composed of 1000 randomly chosen points). The total sensitivity index of the uncontrollable variable $S_{T_{3}}$ is obtained by averaging the dispersion component $Y_{d}$ (with $1 e 6$ randomly chosen points). We can notice the rapid convergence of the predictivity coefficient $Q_{2}$ and the slower convergence of $\mathbb{E}\left(Y_{d}\right)$. The convergence speed for $S_{1}$ and $S_{2}$ computed from the mean component are not shown here but are similar to the one of $Q_{2}$.

From this particular case (low-dimensional but rather complex numerical model due to non linearities and strong interaction), we conclude that a 100 -size sample is sufficient for fitting the joint GAM and for obtaining precise sensitiv- ity indices. Moreover, for the estimation of the total sensitivity index of the uncontrollable variable, using the predictivity coefficient of the mean component is highly recommended (instead of using the dispersion component). With additional experiments, Iooss and Ribatet (2009) have confirmed this result.

In practice, the way to ensure that the convergence has been reached would be to visualize $Q_{2}$ and its confidence interval (by a bootstrap technique for example) by resampling in the learning sample and progressively increasing its size.

\subsection{Application to an hydrogeologic transport code}

The joint approach is now applied to a complex industrial model of radioactive pollutants transport in saturated porous media using the MARTHE computer code (developed by BRGM, France). In the context of an environmental impact study, MARTHE has been applied to a model of strontium $90\left({ }^{90} \mathrm{Sr}\right)$ transport in saturated media for a radwaste temporary storage in Russia (Volkova et al., 2008). Only a partial characterization of the site has been made and, consequently, values of the model input variables are not known precisely: 20 scalar input variables have been considered as random variables, each of them associated to a specified probability density function. The model output variables of interest concern the ${ }^{90} \mathrm{Sr}$ concentration values in different spatial locations. One of the main goals of this study is to identify the most influential variables of the computer code in order to improve the characterization of the site in a judicious way. Because of large computing times of the MARTHE code, the Sobol sensitivity indices are computed using metamodels (boosting regression trees model for Volkova et al., 2008 and Gaussian process model for Marrel et al., 2008).

As a perspective of the Volkova et al. (2008) work, Iooss (2008) studies more precisely the influence of the spatial form of an hydrogeologic layer. The method consists in performing a geostatistical simulation of this layer (which is a two-dimensional spatial random field), before each calculation of the computer model. This geostatistical simulation 
Table 3 Sobol sensitivity indices (with standard deviations) for the Ishigami function: exact and estimated values from simple GAM and simple Gp model. "Method" indicates the estimation method: MC for the Monte-Carlo procedure, Eq for a deduction from the model equations and $Q_{2}$ for the deduction of the predictivity coefficient $Q_{2}$.

\begin{tabular}{|c|c|c|c|c|c|c|c|}
\hline \multirow{2}{*}{ Indices } & \multirow{2}{*}{$\begin{array}{c}\text { Exact } \\
\text { Values }\end{array}$} & \multicolumn{3}{|c|}{ Simple GAM } & \multicolumn{3}{|c|}{ Simple Gp } \\
\hline & & Values & $s d$ & Method & Values & $s d$ & Method \\
\hline$S_{1}$ & 0.314 & 0.333 & $6 e-3$ & MC & 0.292 & $7 e-3$ & MC \\
\hline$S_{2}$ & 0.442 & 0.441 & $6 e-3$ & MC & 0.417 & $7 e-3$ & MC \\
\hline$S_{T_{3}}$ & 0.244 & 0.249 & - & $Q_{2}$ & 0.250 & - & $Q_{2}$ \\
\hline$S_{12}$ & 0 & 0 & - & $\mathrm{Eq}$ & 0.004 & $7 e-3$ & $\mathrm{MC}$ \\
\hline
\end{tabular}
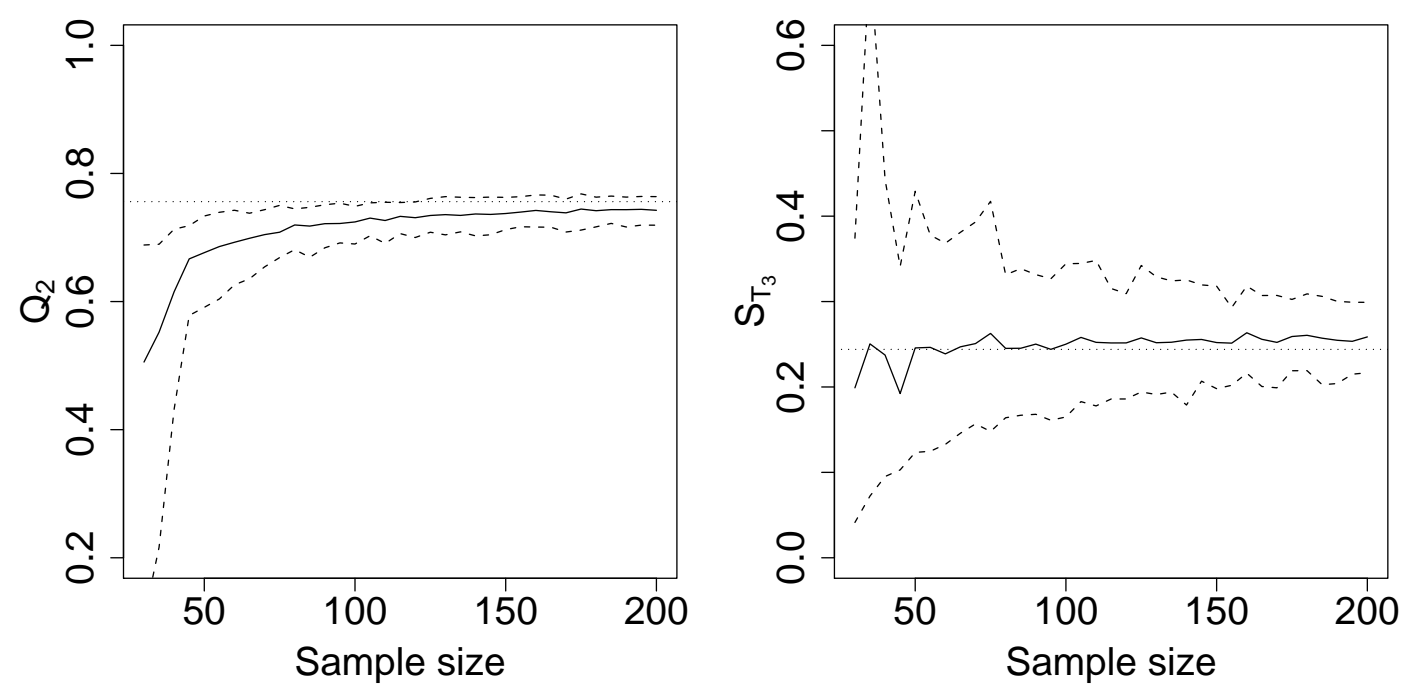

Fig. 4 For the Ishigami function, mean and 90\%-confidence interval (based on 100 replicates) of joint GAM $Q_{2}$ and $S_{T_{3}}$ in function of the learning sample size $n$.

is rather complex and the resulting spatial field cannot be summarized by a few scalar values. Therefore, as explained in our introduction, this hydrogeologic layer form has to be considered as an uncontrollable variable of the computer model. Additionally to the uncontrollable variable, 16 scalar input variables remain uncertain and are treated as random variables. It concerns the permeability of different geological layers, the longitudinal and transversal dispersivity coefficients, the sorption coefficients, the porosity and meteoric water infiltration intensities.

In order to keep coherence with Volkova et al. (2008) previous study, the learning sample size has been chosen to be the same: $N=300$. This size is in adequation with the heuristic recommandation of 10 observations per input dimension (Loeppky et al., 2008; Marrel et al., 2008), used in most of the practical studies on deterministic computer codes. The Latin Hypercube Sampling method is used to obtained a sample of $N$ random vectors (each one of dimension 16). In addition, $N$ independent realizations of the spatial random field (noticed by $\varepsilon$ ) are obtained by a specific geostatistical simulation algorithm (Iooss, 2008). Performing independent realizations for each of the simulator run has been imposed by the small number of available runs (300) relatively to the high-dimensional model (20). Moreover, one of our primary concern was also to perform an uncertainty propagation study, in which replicates have to be avoided. In any case, more interesting designs should be chosen, making replicates for example by changing the controllable input factors while keeping fixed the geostatistical realization. However, such ideas are well beyond the scope of the current paper (see Anderson-Cook et al., 2009, for a recent review about the design issue).

After 8 calculation days, we obtain 300 observations of the output variable of the MARTHE model $\left({ }^{90} \mathrm{Sr}\right.$ concentration at the domain center). As two computer runs have given incoherent values, we keep 298 observations. For the GLMs and GAMs construction phase, the large data dispersion suggests the use of logarithmic link functions for $g$ and $h$ (see Eqs (1) and (2)). Due to the large number of inputs, a manual term selection process has been applied. No interaction term has been found to be explicative in the GLMs. However, a bi-dimensional spline term has been added in the GAMs because of convincing deviance contribution and negligible pvalue. To find this significant interaction term, we have not introduced in the model all the 120 interaction terms. We have sequentially tested all the interaction terms involving one significant first order term ( $k d 1, k d 2$, per2 and per 3$)$ and each other factor. Then, we keep the interaction terms which show some explanatory contribution to the model. 
The results are summarized below by giving the explained deviance and the explanatory terms involved in the formulas:

- Simple GLM: $D_{\text {expl }}=60 \%$ with the terms $k d 1, k d 2$, per 1 , per 2 .

- Joint GLM: $D_{\text {expl }}($ mean $)=66.4 \%$, with the same terms than the simple GLM, $D_{\text {expl }}$ (dispersion) $=8.7 \%$ with the terms $k d 1$ and per 3 .

- Simple GAM: $D_{\text {expl }}=81.8 \%$ with $s(k d 1), s(k d 2), s($ per 3$)$, $s($ per $2, k d 2)$.

- Joint GAM: $D_{\text {expl }}($ mean $)=98.1 \%$ with the same terms than the simple GAM, $D_{\text {expl }}($ dispersion $)=29.7 \%$ with $k d 1, k d 2$.

$k d 1, k d 2$ and per 1, per 2 , per 3 are respectively the sorption coefficients and the permeabilities of the different hydrogeologic layers. One observes that the GAM models outperform the GLM ones. The predictivity coefficient (computed by the leave-one-out method) of the simple GAM gives $Q_{2}=$ $76.4 \%$, while for the simple GLM $Q_{2}=58.8 \%$.

Figure 5 shows the deviance residuals against the fitted values for the joint GLM, simple GAM and joint GAM models. For the joint GLM approach, some outliers are not visible to keep the figure readable. As a consequence, the GAMs clearly lead to smaller residuals. Moreover, the joint GAM outperforms the simple GAM due to the right explanation of the dispersion component. It can be seen that the joint GAM allows to suppress the bias involved by the heteroscedasticity, while simple GAM residuals are affected by this bias.

Figure 6 shows the proportion $\Delta$ of observations that lie within the $\alpha \%$ theoretical confidence interval against the confidence interval $\alpha$. By definition, if a model is suited for both mean and dispersion modelings, the points should be located around the $y=x$ line. As a consequence, this plot is useful to compare the goodness of fit for the different models. It can be seen that the joint GAM is clearly the most accurate model. Indeed, all its points are close to the theoretical $y=x$ line, while the joint GLM (resp. simple GAM) systematically leads to underestimations (resp. overestimations). Consequently, from the Figures 5, 6, one deduces that the joint GAM model is the most competitive one. On one hand, the mean component is modeled accurately without any bias. On the other hand, the dispersion component is competitively modeled leading to reliable confidence intervals.

Table 4 gives the main Sobol sensitivity indices for the joint GLM, joint GAM and simple GAM (using $10^{4}$ model computations for one index estimation). The Sobol indices of the interactions between controllable variables are not given (except between $k d 2$ and per 2 ) because these interactions are not included in the formulas of the two joint models. Therefore, their Sobol indices are zero. The two joint models give similar results for all first order sensitivity indices. The sorption coefficient of the second layer $k d 2$ explained more than $52 \%$ of the output variance, while the permeability of the second layer per 2 explained more than $5 \%$. Some large differences arise in the total influence of the uncontrollable variable $\varepsilon: 38.2 \%$ for the joint GLM and $27.7 \%$ for the joint GAM. Moreover, the joint GLM shows an influence of the interaction between per 3 and $\varepsilon$, while the joint GAM shows an influence of the interaction between $k d 2$ and $\varepsilon$. In this application, we consider the joint GAM results more reliable than the joint GLM ones because the joint GAM captures more efficiently the mean and dispersion components of the data than the joint GLM.

By comparing the joint GAM results with the simple GAM results, some significant differences can be printed out:

- The $k d 1$ first order sensitivity index is overestimated using the simple GAM (14.0\% instead of $3.7 \%$ for the joint GAM). Indeed, the deviance analysis of the joint GAM dispersion component shows a high contribution of $k d 1$, which means that the interaction between $k d 1$ and the uncontrollable variable is probably large. For a standard metamodel, like the simple GAM, this interaction is not found out and leads to a wrong estimation of the first order sensitivity index of $k d 1$.

- For the simple metamodels, using the relation $S_{T}(\varepsilon)=$ $1-Q_{2}$, the total sensitivity index of the uncontrollable variable is underestimated: $23.5 \%$ (simple GAM) instead of $27.7 \%$ (joint GAM). The classical metamodels tend to explain some parts of the data which can be adequately included in the dispersion component of the joint GAM during the iterative fitting algorithm.

- Contrary to the other metamodels, the joint GAM allows to prove that only $k d 1$ and $k d 2$ interact with the uncontrollable variable.

As a conclusion, these sensitivity analysis results will be very useful to the physicist or the modeling engineer during the model construction and calibration steps. In this specific application, the sensitivity analysis shows that the geometry of the second hydrogeological layer has a strong influence (up to $28 \%$ ) on the predicted ${ }^{90} \mathrm{Sr}$ concentration. Therefore, an accurate modeling of this geometry, coupled with a better knowledge of the most influential variable $k d 2$, are the key steps to an important reduction of the model prediction uncertainties.

\section{Conclusion}

This paper has proposed a solution to compute variancebased sensitivity indices of stochastic computer model outputs. It consists in modeling the mean and the dispersion of the code outputs by two explanatory models. The classical way is to separately build these models. In this paper, the use of the joint modeling is preferred. This the- 

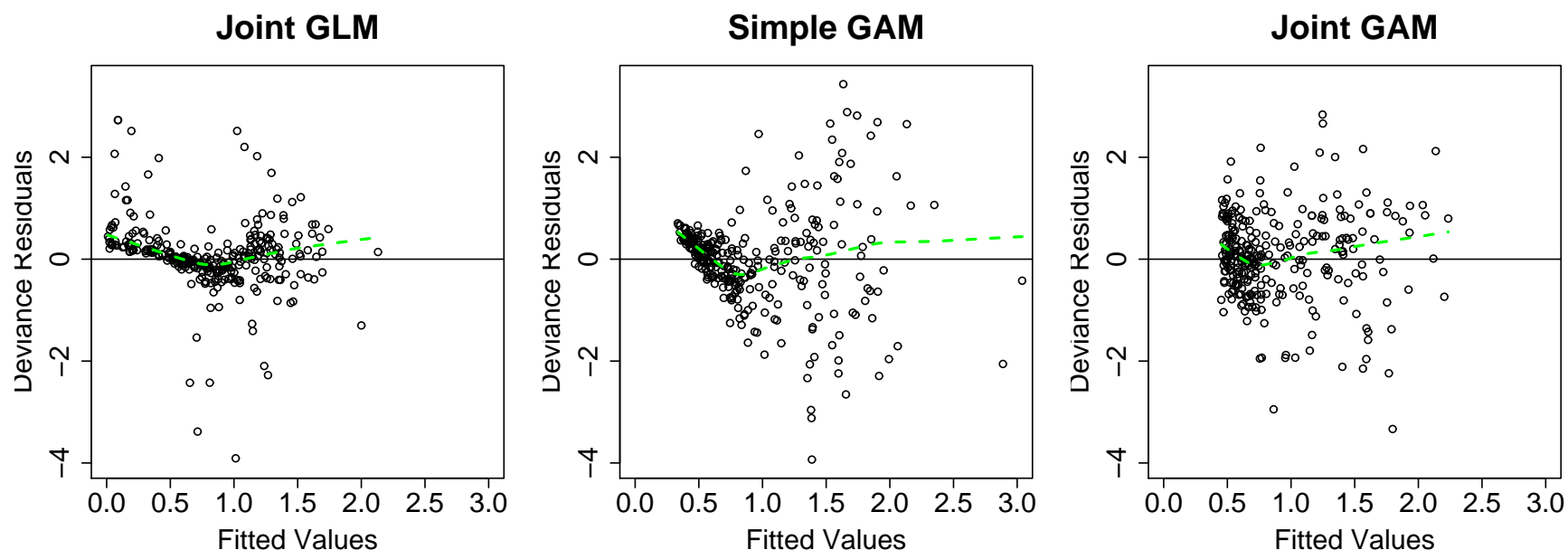

Fig. 5 Deviance residuals (mean component) for the Simple GAM, Joint GAM and Joint GLM versus the fitted values (MARTHE application). Dashed lines correspond to local polynomial smoothers.

Joint GLM

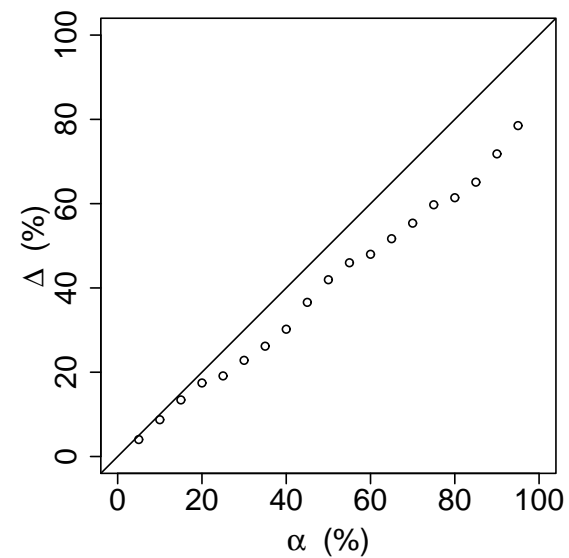

Simple GAM

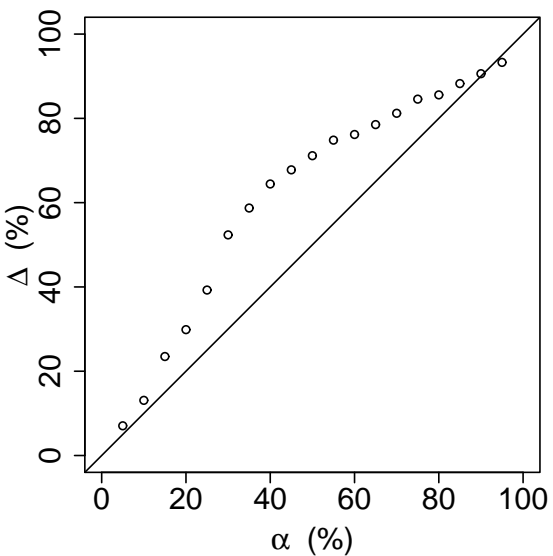

Joint GAM

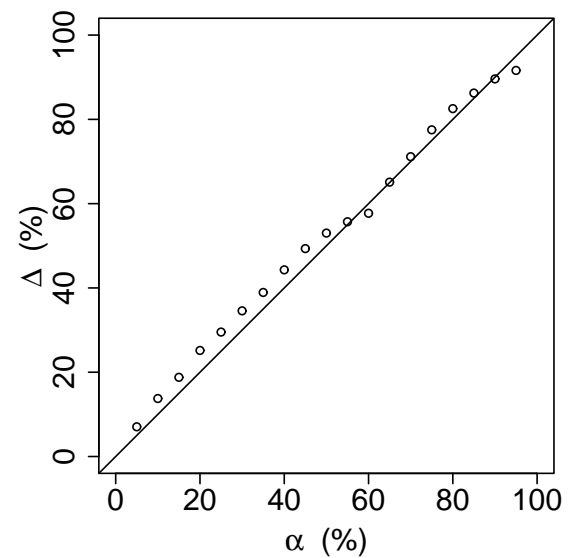

Fig. 6 Proportion $\Delta$ (in percent) of observation that lie within the $\alpha \%$ theoretical confidence interval in function of the confidence level $\alpha$. MARTHE application.

ory, proposed by Pregibon (1984) and Smyth (1989), then extensively developed by Nelder (1998), is a powerful tool to fit the mean and dispersion components simultaneously. Zabalza et al. (1998) already applied this approach to model stochastic computer code. However, the behavior of some numerical models can be highly complex and non linear. In the present paper, some examples show the limit of this parametric joint model. Being inspired by Rigby and Stasinopoulos (1996) who use non parametric joint additive models (restricted to Gaussian cases), we have developed a more general joint model using GAMs and quasi distributions. Like GLMs, GAMs are a suited framework because it allows variable and model selections via quasi-likelihood function, classical statistical tests on coefficients and graphical displays. Additional works using joint GLMs and joint GAMs for computer experiments can be found in Iooss and Ribatet (2009).

The joint GAM has proven its flexibility to fit complex data: we have obtained the same performance for its mean and dispersion components as the powerful Gp model. Deal- ing with computer codes involving many factors and strong interactions between model factors, it would be convenient to look more precisely at other joint models, as the joint $\mathrm{Gp}$ model we have shortly described and used. An analytic case on the Ishigami function shows that these two non parametric joint models (GAM and $\mathrm{Gp}$ ) are adapted to complex heteroscedastic situations where classical metamodels are inadequate. Moreover, it offers a theoretical basis to compute Sobol sensitivity indices in an efficient way. The analytical formulas available with the joint GAM are very useful to complete the sensitivity analysis results and to improve our model understanding and knowledge.

The performance of the joint model approach was assessed on an industrial application. Compared to other methods, the modeling of the dispersion component allows to obtain a robust estimation of the total sensitivity index of the uncontrollable variable, which leads to correct estimations of the first order indices of the controllable variables. In addition, it reveals the influential interactions between the un- 
Table 4 Estimated Sobol sensitivity indices (with standard deviations obtained by 100 repetitions) for the MARTHE code. "Method" indicates the estimation method: MC for the Monte-Carlo procedure, Eq for a deduction from the model equations and $Q_{2}$ for the deduction of the predictivity coefficient $Q_{2}$. "—" indicates that the value is not available.

\begin{tabular}{|c|c|c|c|c|c|c|c|c|c|}
\hline \multirow{2}{*}{ Indices } & \multicolumn{3}{|c|}{ Joint GLM } & \multicolumn{3}{|c|}{ Joint GAM } & \multicolumn{3}{|c|}{ Simple GAM } \\
\hline & Values & $s d$ & Method & Values & $s d$ & Method & Values & $s d$ & Method \\
\hline$S(\mathrm{kd} 1)$ & 0.002 & $0.6 \mathrm{e}-2$ & MC & 0.037 & $1.0 \mathrm{e}-2$ & MC & 0.140 & $1.0 \mathrm{e}-2$ & MC \\
\hline$S(\mathrm{kd} 2)$ & 0.522 & $0.6 e-2$ & MC & 0.524 & $1.0 \mathrm{e}-2$ & $\mathrm{MC}$ & 0.550 & $1.1 \mathrm{e}-2$ & $\mathrm{MC}$ \\
\hline$S($ per1) & 0.018 & $0.7 e-2$ & MC & 0 & - & $\mathrm{Eq}$ & 0 & - & $\mathrm{Eq}$ \\
\hline$S($ per2) & 0.052 & $0.6 e-2$ & $\mathrm{MC}$ & 0.078 & $1.0 \mathrm{e}-2$ & $\mathrm{MC}$ & 0.044 & $1.0 \mathrm{e}-2$ & $\mathrm{MC}$ \\
\hline$S($ per3) & 0 & - & $\mathrm{Eq}$ & 0.005 & $1.0 \mathrm{e}-2$ & $\mathrm{MC}$ & 0.008 & $1.0 \mathrm{e}-2$ & $\mathrm{MC}$ \\
\hline$S(\mathrm{kd} 2$,per2) & 0 & - & $\mathrm{Eq}$ & 0.063 & $1.0 \mathrm{e}-2$ & $\mathrm{MC}$ & 0.026 & $1.0 \mathrm{e}-2$ & $\mathrm{MC}$ \\
\hline$S_{T}(\varepsilon)$ & 0.382 & $0.2 \mathrm{e}-2$ & MC & 0.277 & $0.3 e-2$ & $\mathrm{MC}$ & 0.235 & - & $Q_{2}$ \\
\hline$S(\mathrm{kd} 1, \varepsilon)$ & ] $0,0.382]$ & - & $\mathrm{Eq}$ & ] $0,0.277]$ & - & $\mathrm{Eq}$ & - & - & - \\
\hline$S(\mathrm{kd} 2, \varepsilon)$ & 0 & - & $\mathrm{Eq}$ & $0,0.277]$ & - & $\mathrm{Eq}$ & - & - & - \\
\hline$S($ per1 $1, \varepsilon)$ & 0 & - & $\mathrm{Eq}$ & 0 & - & $\mathrm{Eq}$ & - & - & - \\
\hline$S($ per $2, \varepsilon)$ & 0 & - & $\mathrm{Eq}$ & 0 & - & $\mathrm{Eq}$ & - & - & - \\
\hline$S($ per $3, \varepsilon)$ & ] $0,0.382]$ & - & $\mathrm{Eq}$ & 0 & - & $\mathrm{Eq}$ & - & - & - \\
\hline
\end{tabular}

controllable variable and the other input variables. Obtaining quantitative values for these interaction effects is still an open issue, but a challenging problem. Finally, the joint model would also serve in the uncertainty propagation studies of complex models, to obtain the full distribution of the model output.

In the whole, all statistical analysis were performed using the $\mathrm{R}$ software environment $\mathrm{R}$ Development Core Team, 2006). In particular, the following functions and packages were useful: the "glm" function to fit a simple GLM, the "mgcv" (Multiple Smoothing Parameter Estimation by GCV) package to fit a simple GAM, and the "sensitivity" package to compute Sobol indices. We also developed the "JointModeling" package to fit joint models (including joint GLM and joint GAM).

\section{References}

Anderson-Cook, C., Borror, C., and Montgomery, D. (2009). Response surface design evaluation and comparison. Journal of Statistical Planning and Inference, 139:629-641.

Bates, R., Kenett, R., Steinberg, D., and Wynn, H. (2006). Achieving robust design from computer simulations. Quality Technology and Quantitative Management, 3:161-177.

Bayarii, M., Berger, J., Cafeo, J., Garcia-Donato, G., Liu, F., Palomo, J., R.J.Parthasarathy, Paulo, R., Sacks, J., and Walsh, D. (2007). Computer model validation with functional output. The Annals of Statistics, 35:1874-1906.

Boukouvalas, A. and Cornford, D. (2009). Learning heteroscedastic Gaussian processes for complex datasets. Technical report, Neural Computing Research Group, Aston University.

Bursztyn, D. and Steinberg, D. (2006). Screening experiments for dispersion effects. In Dean, A. and Lewis, S., editors, Screening - Methods for experimentation in industry, drug discovery and genetics. Springer.
Chen, V., Tsui, K.-L., Barton, R., and Meckesheimer, M. (2006). A review on design, modeling and applications of computer experiments. IIE Transactions, 38:273-291.

De Rocquigny, E., Devictor, N., and Tarantola, S., editors (2008). Uncertainty in industrial practice. Wiley.

Fang, K.-T., Li, R., and Sudjianto, A. (2006). Design and modeling for computer experiments. Chapman \& Hall/CRC.

Ginsbourger, D., Roustant, O., and Richet, Y. (2008). Kriging with heterogeneous nugget effect for the approximation of noisy simulators with tunable fidelity. In Proceedings of Joint Meeting of the Statistical Society of Canada and the Socit Franaise de Statistique, Ottawa, Canada.

Hastie, T. and Tibshirani, R. (1990). Generalized additive models. Chapman and Hall, London.

Helton, J., Johnson, J., Salaberry, C., and Storlie, C. (2006). Survey of sampling-based methods for uncertainty and sensitivity analysis. Reliability Engineering and System Safety, 91:1175-1209.

Homma, T. and Saltelli, A. (1996). Importance measures in global sensitivity analysis of non linear models. Reliability Engineering and System Safety, 52:1-17.

Iooss, B. (2008). Treatment of spatially dependent variables in sensitivity analysis of model output methods. Note Technique CEA/DEN/CAD/DER/SESI/LCFR/NT DO 4 06/03/08, CEA, Cadarache, France.

Iooss, B., Lhuillier, C., and Jeanneau, H. (2002). Numerical simulation of transit-time ultrasonic flowmeters due to flow profile and fluid turbulence. Ultrasonics, 40:10091015.

Iooss, B. and Ribatet, M. (2006). Analyse de sensibilité globale de modèles numériques à paramètres incontrôlables. In Proceedings of 38èmes Journées de Statistique, Clamart, France.

Iooss, B. and Ribatet, M. (2009). Global sensitivity analysis of computer models with functional inputs. Reliability Engineering and System Safety, 94:1194-1204.

Juutilainen, I. and Röning, J. (2005). A comparaison of methods for joint modelling of mean and dispersion. In 
Proceedings of the 11th Symposium on ASMDA, Brest, France.

Kennedy, M. and O'Hagan, A. (2001). Bayesian calibration of computer models. Journal of the Royal Statistical Society, 63(3):425-464.

Kleijnen, J. (1997). Sensitivity analysis and related analyses: a review of some statistical techniques. Journal of Statistical Computation and Simulation, 57:111-142.

Kleijnen, J. and van Beers, W. (2005). Robustness of kriging when interpolating in random simulation with heterogeneous variances: some experiments. European Journal of Operational Research, 165:826-834.

Lee, Y. and Nelder, J. (2003). Robust design via generalized linear models. Journal of Quality Technology, 35(1):212.

Loeppky, J., Sacks, J., and Welch, W. (2008). Choosing the sample size of a computer experiment: A practical guide. Technical Report 170, National Institute of Statistical Sciences.

Marrel, A., Iooss, B., Van Dorpe, F., and Volkova, E. (2008). An efficient methodology for modeling complex computer codes with Gaussian processes. Computational Statistics and Data Analysis, 52:4731-4744.

McCullagh, P. and Nelder, J. (1989). Generalized linear models. Chapman \& Hall.

Nelder, J. (1998). A large class of models derived from generalized linear models. Statistics in Medicine, 17:27472753.

Nelder, J. and Pregibon, D. (1987). An extended quasilikelihood function. Biometrika, 74:221-232.

Nelder, J. and Wedderburn, R. (1972). Generalized linear models. Journal of the Royal Statistical Society A, 135:370-384.

Phadke, M. (1989). Quality engineering using robust design. Prentice-Hall, New-York, NY.

Pregibon, D. (1984). Review of "Generalized Linear Models" by McCullagh and Nelder. Annals of Statistics, 12:1589-1596.

R Development Core Team (2006). R: A Language and Environment for Statistical Computing. ISBN 3-900051-070 , Vienna, Austria.

Rigby, R. and Stasinopoulos, D. (1996). A semi-parametric additive model for variance heterogeneity. Statistics and Computing, 6:57-65.

Sacks, J., Welch, W., Mitchell, T., and Wynn, H. (1989). Design and analysis of computer experiments. Statistical Science, 4:409-435.

Saltelli, A., Chan, K., and Scott, E., editors (2000). Sensitivity analysis. Wiley Series in Probability and Statistics. Wiley.

Smyth, G. (1989). Generalized linear models with varying dispersion. Journal of the Royal Statistical Society $B, 51: 47-60$.
Sobol, I. (1993). Sensitivity estimates for non linear mathematical models. Mathematical Modelling and Computational Experiments, 1:407-414.

Vining, G. and Myers, R. (1990). Combining Taguchi and response-surface philosophies - a dual response approach. Journal of Quality Technology, 22:38-45.

Volkova, E., Iooss, B., and Van Dorpe, F. (2008). Global sensitivity analysis for a numerical model of radionuclide migration from the RRC "Kurchatov Institute" radwaste disposal site. Stochastic Environmental Research and Risk Assesment, 22:17-31.

Wood, S. and Augustin, N. (2002). GAMs with integrated model selection using penalized regression splines and applications to environmental modelling. Ecological modelling, 157:157-177.

Zabalza, I., Dejean, J., and Collombier, D. (1998). Prediction and density estimation of a horizontal well productivity index using generalized linear models. In ECMOR VI, Peebles. 